\title{
A Gateway Selection Mechanism for Internet-assisted MANETS
}

\author{
https://doi.org/10.3991/ijoe.v17i13.24335 \\ Le Anh Ngoc ${ }^{1}$, Nguyen Duy Tan ${ }^{2}$, Quy Vu Khanh ${ }^{2(\varpi)}$ \\ ${ }^{1}$ Swinburne Vietnam, FPT University, Vietnam \\ ${ }^{2}$ Hung Yen University of Technology and Education, Hungyen, Vietnam \\ quyvk@utehy.edu.vn
}

\begin{abstract}
Internet-assisted MANETs architecture is an emerging solution, strongly introduced in recent years. In fact, MANETs are self-configurable wireless network models for convenient communication between mobile nodes in the network. Because of simplicity, MANETs are applied in a series of domains to serve humanity, such as intelligent agriculture, intelligent transport system, and IoT ecosystem. To extend the ability of MANETs, the integration of Internet and MANETs to form Internet-assisted MANETs architecture was introduced in many studies. However, one of the biggest problems of this solution is how to choose the optimal Internet gateway. This study proposes a gateway selection mechanism based on parameters, including hop number and gateway traffic. To evaluate the effectiveness of the proposed mechanism, we compare the proposed solution with previous solutions. The simulation results demonstrated that our solution improved end-to-end delay, routing overload, and loss packet ratio compared to previous protocols.
\end{abstract}

Keywords - internet gateway, internet-assisted MANET, performance improvement

\section{Introduction}

Mobile Ad Hoc Network (MANETs) is a network organization that can self-install and configure parameters to communicate within the network without relying on existing network infrastructure. The most vital features of MANETs are fast deployment and self-configuration. Thanks to these characteristics, the MANET has been used in various fields serving humanity, such as smart healthcare, smart transportation system, disaster recovery, and IoT ecosystems [1-2]. To extend the ability of MANETs, the integration of Internet and MANETs to form Internet-assisted MANETs architecture, in Figure 1 has been introduced [3]. The combination of the Internet and MANET that introduces unprecedented capabilities to MANETs that has been demonstrated in many studies [4-5]. However, the solution also faces challenges such as connectivity loss and energy efficiency [6], security, and performance improvement [7-8]. 


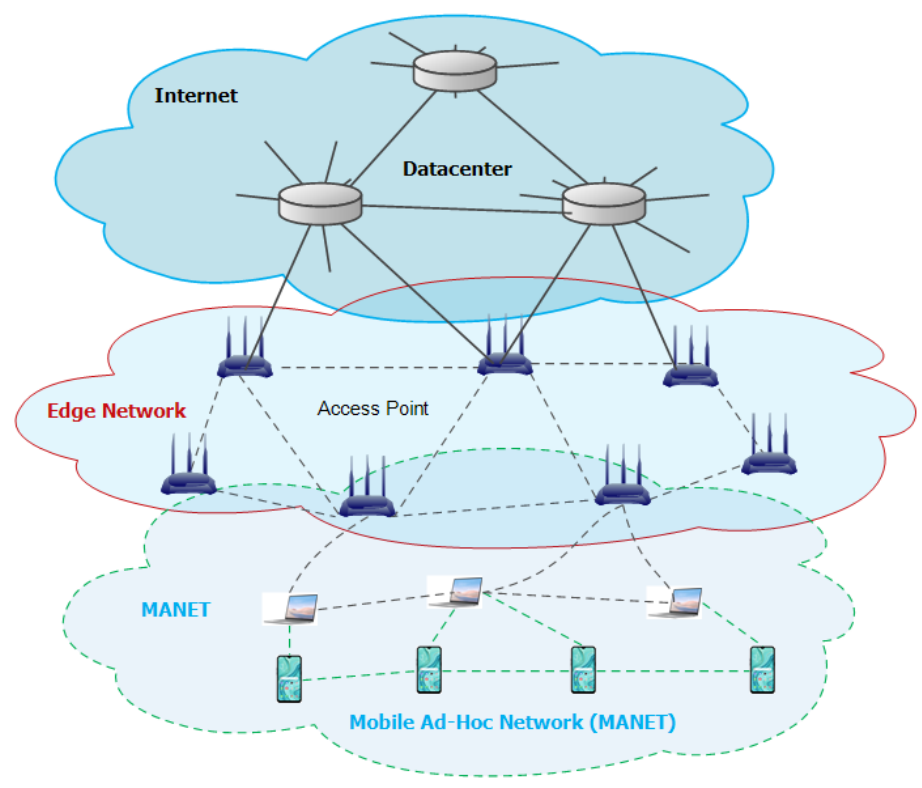

Fig. 1. An architecture for Internet-assisted MANET

To improve the performance of the Internet-assisted MANETs, one of the main problems is how to choose an Internet gateway [9]. There can be more than one gateway to communicate between the Internet and MANETs in the Internet-assisted MANET achitecture. When a mobile node of MANETs receives the advertisement messages from many Internet gateways, it needs to select a suitable gateway.

The traditional gateway selection mechanisms [10-11] use the distance (hop number) to determine a gateway without attention to gateway traffic. As a result, the gateway that has the minimum hop numbers will be selected. In most scenarios, this solution led to all communication traffic between MANETs and the Internet focusing on this gateway and causing a bottleneck status, reducing system performance. In reality, the Internet gateway selection problem should trade-off between distance (hop number) and gateway traffic.

To solve the problem, in this study, we propose a traffic-based Internet gateway selection mechanism. The rest of the paper is organized as follows: In section 2, we presented the related works. In section 3, the proposed mechanism is described. Simulation results and analysis are presented in section 4, and section 5 is the conclusion and further research direction.

\section{Internet-assisted MANET architecture}

A typical architecture of Internet-assisted MANET is shown in Figure 1. It consists of three layers. Layer 3 is the mobile node of the MANET network. In Layer 3, the mobile nodes operate based on the typical routing protocols such as AODV, DSR, 
OLSR, etc. Besides, the mobile nodes can self-configure network parameters to communicate without relying on pre-installed network infrastructures. In our proposed model, to use applications and services on the Internet, a mobile node of MANET has installed the AODV routing protocol and is connected to Internet gateways.

An Internet gateway is a particular network node in the second layer of the proposed architecture. In reality, gateway nodes can communicate with both MANET and the Internet. Each gateway has a different processing capacity (including bandwidth, buffering, traffic, etc.). The primary responsibility of the gateway is to transfer the traffic flow between the Internet and MANET. In the Internet-assisted MANET structure, we always assume more than one gateway.

In layer 1 of the proposed architecture is the Internet layer. The Internet is a set of data servers (datacenter) with strong capacity and ultra-high processing speed, ultrawide bandwidth to provide services and applications to the under layers.

In this study, for the purpose to propose gateway selection solutions, we present a simplified architecture as shown in Figure 2. This architecture consists of 2 layers: Gateway and MANET. To be able to communicate to an Internet gateway, there are two mechanisms:

1. Periodically, the gateway nodes advertise GWADV packets (Gateway Advertisement) to MANET

2. When a mobile node needs to communicate to a gateway, it sends a gateway discovery message. Then, based on the received routing information, it will update into the routing table for communication.

Note that the existing mechanisms use the distance (hop number) to making-decision a gateway. However, our approach uses a combined metric, including hop number and gateway traffic, to calculate an optimal Internet gateway.

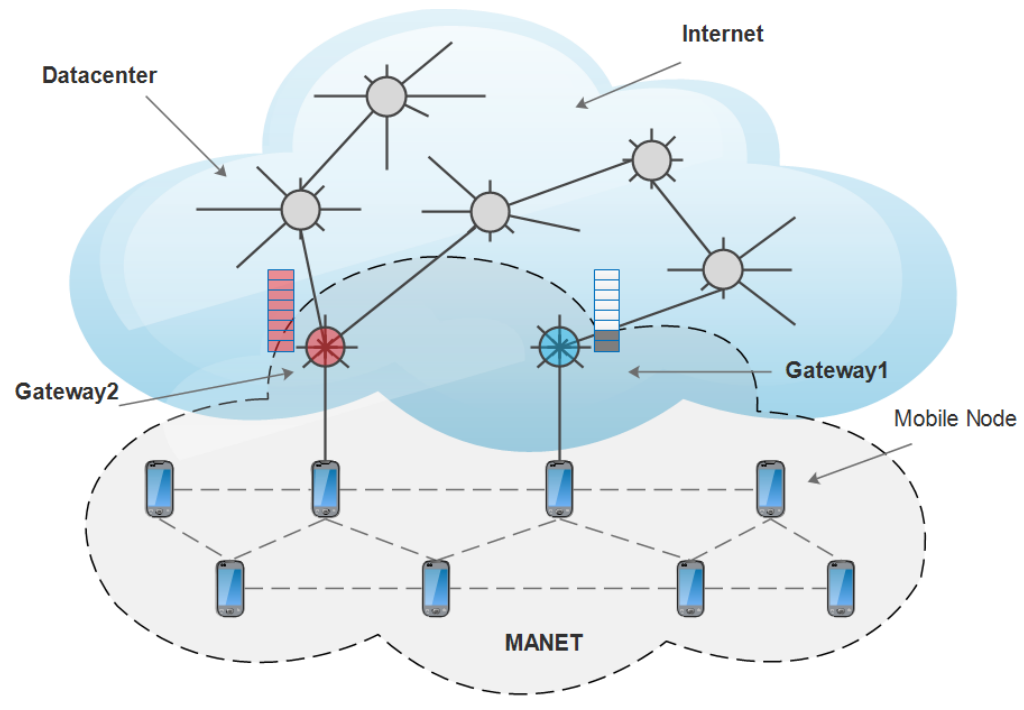

Fig. 2. The Internet-assisted MANET Architecture 


\section{The problem}

An Internet gateway (IGW) is a unique network node to support access to the services and applications from MANET to the Internet. It relays the network traffics from MANET to the Internet and vice versa. Therefore, the gateway is a component of both the Internet and MANET. In practice, a gateway has multiple network interfaces, each of which connects to a different network. In Internet-assisted MANET architecture, a gateway has two network interfaces. The wireless interface joins to MANET, and the remaining wire interface connects to the Internet.

As discussed above, the most traditional strategies are based on the smallest hop number to choose an appropriate gateway. In some scenarios, all mobile nodes connect to this gateway to the Internet. Consequently, this gateway is overloaded, and it becomes a bottleneck node. In reality, besides the distance from the mobile node to a MANET is a convenient solution for communication between mobile nodes inside the wireless network. gateway, a vital parameter is the traffic of gateways. To solve this problem, we propose a new gateway selection mechanism. Our proposed solution is based on two parameters, the hop number and traffic (gateway traffic). According to our approach, Internet gateway periodically broadcasts GWADV messages (Gateway Advertiser). The mobile nodes update routing information and exchange it with each other node. The outstanding features of the proposed protocol are discussed in the following sections.

\section{$4 \quad$ Related work}

MANET is a convenient solution for communication between mobile nodes inside the wireless network. The network nodes in MANET are personal mobile devices that have limited memory, battery, and processing capabilities. Besides, due to the mobility characteristic of the mobile nodes, the network structure is frequently changed. To enhance the ability of MANET, the combination MANET and Internet has been introduced. This requires an Internet gateway selection algorithm for communication between MANET and the Internet, as follows.

In [10], authors proposed an Internet gateway selection solution based on distance (hop number) to communicate between the ad-hoc network and the Internet. Similarly, in [11], the authors presented a gateway discovery algorithm to communicate between MANET and Internet. A common feature of traditional Internet gateway selection solutions is to use the distance (hop number) as a metric. The advantage of this solution is the simple algorithm. The disadvantage is the communication traffic between the Internet and MANET will concentrate through the gateway with minimal hop number. In many scenarios, it will result in local network congestion.

Survey results [12-13] showed that, in recent times, the optimal Internet gateway selection solutions had attracted researchers, many modern solutions had been proposed, as follows:

In [14], the authors proposed a schema to select an Internet gateway for communication between MANET and Internet. The solution allows an Internet gateway selection 
based on the network status. Simulation results demonstrated that the proposed algorithm enhanced the performance parameters compared to the existing traditional algorithm. In [15], the authors introduced a solution to select an Internet gateway based on game theory to load balancing among gateways and enhance the performance parameters for Internet-MANET networks. In [16], the authors introduced a mechanism to select an Internet gateway for communication in the heterogeneous wireless network, include satellite, UAVs and ground devices. The solution obtains multi-metrics from cross-layer and combine them in the cost function to choose the optimal gateway. Simulation results showed that the proposed solution enhanced the performance parameters compared with the existing solution. In [17], authors introduced a saving energy solution to select an Internet gateway for communication between MANET and Internet gateway. The study used the multi-interface collaboration mechanism based on ZigBee radio. Simulation result demonstrated that the proposed solution enhanced the performance parameters and saving energy compared with the existing solutions. In [18], the authors introduced a model to enable access to the multithreaded Internet gateway for communication between the ad-hoc network and the Internet. Simulation results demonstrated that the proposed solution enhanced the performance parameters, saving energy compared to the existing solution.

Analysis of recent related proposals of this domain showed the state-of-the-art Internet gateway selection proposals have a common framework. It constrains many parameters in the cost function to make decisions on optimal gateway selection.

Surveyed results also show that in order to support QoS requirements for the services and applications, many parameters are used, such as hop number, gateway traffic, the queue length of the interface, and multi-interface collaboration. These proposed solutions have initially improved the network performance, load balancing between gateways. However, many parameters are taken into the making-decision to select an optimal gateway that will reduce the flexibility that is an advantage of MANET and increase energy consumption and latency.

Accordingly, our opinion, the selection parameters should trade-off between QoS and energy efficiency. Therefore, in this study, we proposed a solution that take the gateway traffic parameter into the cost function to select an Internet gateway for Internet-assisted MANET. The detailed mechanism is presented in the following section.

\section{$5 \quad$ Proposed solution}

In this section, we present the Internet gateway selection algorithm, which uses parameters such as hop number and traffic of gateway to select an optimal gateway, as follows:

\subsection{Gateway selection routing algorithm}

To limit the overload of the Internet gateway in Internet-assisted MANETs which have multiple Internet gateways, we propose a gateway selection algorithm for the load 
balancing of gateways to improve the performance of Internet-assisted MANET architectures, as follows:

- Step 1: Periodically, gateways will self-introduce by broadcasting Gateway Advertisement messages (GWADV) to MANET with modified header as follows: \{Traffic, Advertisment Header\}. Along with the modified GWADV message, the routing table of a mobile node is also modified to store the Traffic field, as in Figure 3.

To limit broadcasting of Gateway Advertisement packets throughout the network. The traditional solutions set a static value for the TTL (Time to Live) field in the header of the GWADC packet. If $T T L=0$, the GWADV message will not be sent. Then the mobile node will update into the routing table a default Internet gateway.

- Step 2: A mobile node needs to communicate with the Internet. It uses the default gateway stored in the routing table. If the routing table without a default gateway, it will broadcast RREQ messages to discover an Internet gateway. A gateway node or a mobile node that had a gateway entry will send gateway information to the source node with header $\{$ Traffic, RREP AODV $\}$.

- Step 3: After the source node receives gateway information, a gateway set is determined as follows:

$$
\text { Gateway_Set }=\left\{\begin{array}{c}
\text { Gateway }_{1}\left(\text { Hopnumber }_{1}, \text { Traffic }_{1}\right) \\
\text { Gateway }_{2}\left(\text { Hopnumber }_{2}, \text { Traffic }_{2}\right) \\
\vdots \\
\text { Gateway }_{n}\left(\text { Huonumber }_{n}, \text { Traffic }_{n}\right)
\end{array}\right.
$$

Then, the wireless source node will select a suitable Internet gateway based on following metric:

$$
\text { Metric }_{i}=\left\{\begin{array}{c}
\alpha \times \text { Hopnumber }_{i}+\beta \times \text { Traffic }_{i} \\
\alpha+\beta=1
\end{array}\right.
$$

We demonstrate the operating principles as follows: First, a gateway inserts the traffic information into every gateway advertisement message (GWADV) and sends broadcast on the wireless network. A mobile wireless node obtains the GWADV message; it uses Eq. (2) to calculate and determine the Metric $c_{i}$. Then it compares this obtained value with the previous value in the routing table. If the new measure value is better

\begin{tabular}{|c|c|c|c|}
\hline Type & Reserved & Prefix Size & HopCount \\
\hline \multicolumn{4}{|c|}{ Broadcast ID } \\
\hline \multicolumn{4}{|c|}{ Destination IP Address } \\
\hline \multicolumn{4}{|c|}{ Destination Sequence Number } \\
\hline \multicolumn{4}{|c|}{ Source IP Address } \\
\hline \multicolumn{4}{|c|}{ TTL } \\
\hline \multicolumn{4}{|c|}{ Timestamp } \\
\hline \multicolumn{4}{|c|}{ Traffic } \\
\hline
\end{tabular}
than the previous one, the new gateway will be updated into the routing table.

Fig. 3. Modified Gateway Advertisement message format. 


\section{$6 \quad$ Results and analytics}

Our proposed scheme is integrated into the AODV protocol, namely the GatewayAODV protocol. Then we set up simulations on NS2 simulation software to compare the efficiency of Gateway-AODV, AODV, and DSR based on end-to-end delay, packet delivery ratio, and routing load. The working principle of the Gateway-AODV protocol is introduced in Section 2.

In all simulation scenarios, we set up $\alpha=0.8, \beta=0.2$, and the $T T L=3$ in the GWADV messages. In our opinion, the TTL value should be dynamically determined based on the gateway status. As a consequence, the mobile nodes in the broadcast range will obtain the Internet gateway information. This problem will be discussed in our further study.

\subsection{Simulation parameters}

The simulation scenarios are installed consisting of 100 mobile nodes, randomly arranged in a square: $2.000 \times 2.000\left(\mathrm{~m}^{2}\right)$ and five gateway nodes. The movement speed of the mobile node is in the range $[1-10](\mathrm{m} / \mathrm{s})$. Simulations used the random waypoint model; the traffics type is CBR, and the simulation time is 600 (s). The packet size is 512 (bytes). The simulation parameters are shown in Table 1.

Table 1. Simulation Parameters

\begin{tabular}{|l|c|}
\hline Parameters & Values \\
\hline Simulation time & $600(\mathrm{~s})$ \\
\hline Topology & $2000 \times 2000(\mathrm{~m})$ \\
\hline Transmission Range & $250(\mathrm{~m})$ \\
\hline Traffic Type & CBR \\
\hline Number of Mobile Nodes & 100 \\
\hline Movement Speed of Nodes & {$[1-10](\mathrm{m} / \mathrm{s})$} \\
\hline Number of Traffic Sources & 05 \\
\hline Number of Gateways & 05 \\
\hline Mobility Model & Random Waypoint Model \\
\hline Routing Protocol & Gateway-AODV, AODV and DSR \\
\hline
\end{tabular}

\subsection{Performance metrics}

To evaluate the performance of the proposed solution, in this study, we defined the performance metrics as follows:

- Packet Delivery Ratio: This parameter reflects the ratio of the transmitted packet number successfully and the sent data packet number sent by all the traffic sources.

- Average Delay: This parameter reflects the average period to transmit a data packet from the source node to the destination node.

- Routing Load: This parameter reflects the ratio of the control packet number and the total of the sent data packet number. 


\subsection{Results discussion}

The simulation data showed the effectiveness of the proposed approach compared with the traditional approach. The observed data in Figure 4 indicated that the packet delivery ratio of the Gateway-AODV protocol improved by over $10 \%$ than the conventional routing protocols. In particular, when the movement speed of the mobile nodes increases to above $5(\mathrm{~m} / \mathrm{s})$, the improvement is even more apparent.

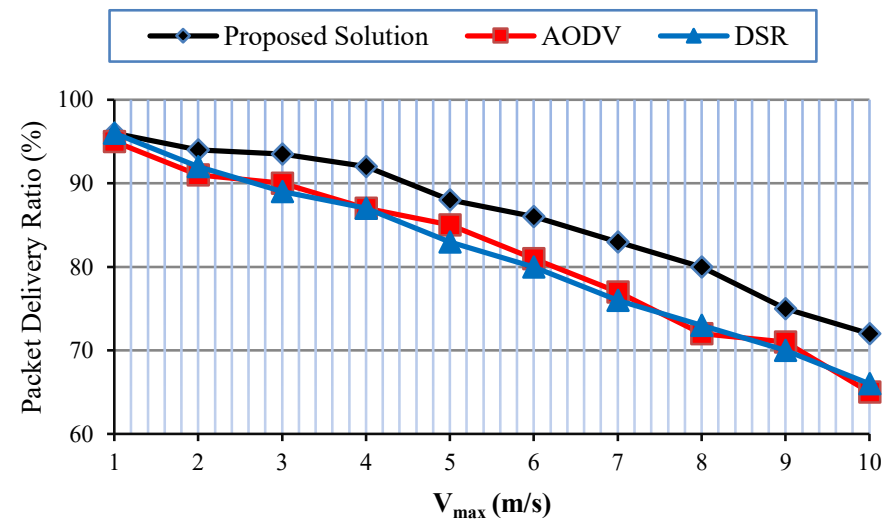

Fig. 4. Packet Delivery Ratio \& Movement Speed of Node

The results in Figure 5 showed that the proposed solution has significantly improved the end-to-end delay compared to traditional solutions. Besides, the results in Figure 6 also showed that the routing load is significantly improved. The achievement of these results thanks to the proposed gateway selection mechanism. Our mechanism not only selects high-bandwidth Internet gateways but also can load balancing between Internet gateways based on the state of these gateways.

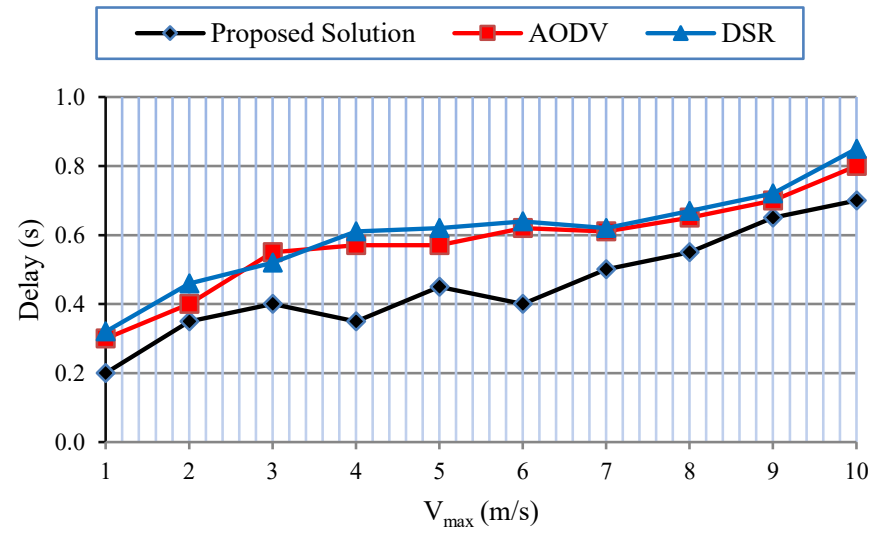

Fig. 5. Delay \& Movement Speed of Node 


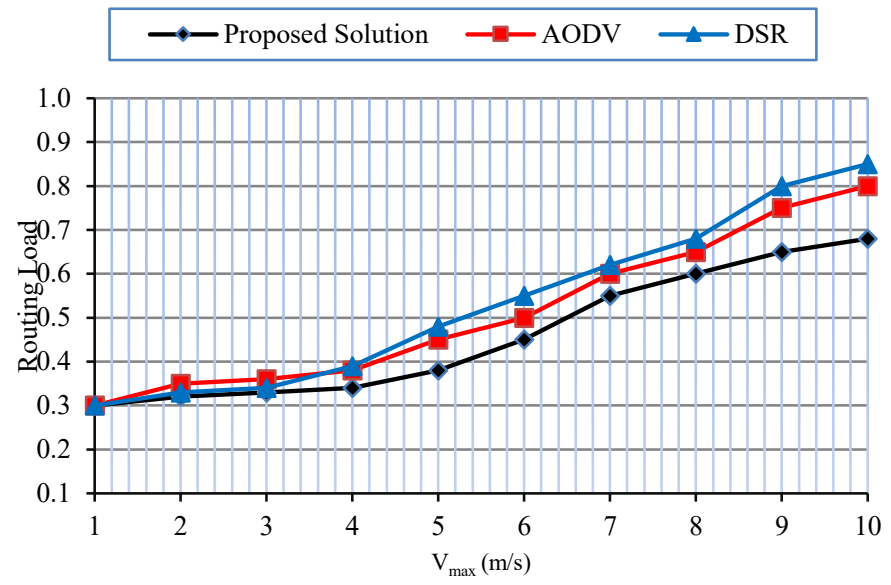

Fig. 6. Routing Load \& Movement Speed of Nodes

\section{$7 \quad$ Conclusion and further research direction}

Integrating the mobile ad-hoc network (MANET) with the Internet introduces unprecedented abilities of MANET have been demonstrated in many services and applications. However, this solution also faces challenges. One of the biggest problems is the optimal Internet gateway selection mechanism. In this paper, a hybrid architecture for communication between MANET and the Internet through Internet gateways has been introduced. Moreover, we have proposed a gateway selection mechanism based on the load balancing among the Internet gateway. The simulation results show that our proposed solution improves performance parameters significantly compared to traditional solutions. In our further research, to limit flooding of the gateway advertisement packets on MANET, routing protocols should set up dynamically broadcast distance based on the capability of the Internet gateways (caching, capacity, etc.) to provide more excellent performance for the Internet-assisted MANET architecture.

\section{Author contributions}

We have conducted the research, analyzed the data, and performed simulations together. All authors had approved the final version. Corresponding Author is Vu Khanh Quy.

\section{$9 \quad$ References}

[1] Deng, L., Liu, F., Zhang, Y. and Wong, W.S., Delay-Constrained Topology-Transparent Distributed Scheduling for MANETs. IEEE Transactions on Vehicular Technology, 2021. 70(1): pp. 1083-1088. https://doi.org/10.1109/TVT.2020.3046856 
Paper-A Gateway Selection Mechanism for Internet-assisted MANETs

[2] Quy, V.K, Ngoc, A.L., An Energy-Efficient Routing Protocol for MANET in Internet of Things Environment. International Journal of Online and Biomedical Engineering, 2021. 17(07):88-99. https://doi.org/10.3991/ijoe.v17i07.23273

[3] Niu, Z., Li, Q., Ma, C., Li, H., Shan, H. and Yang, F., Identification of Critical Nodes for Enhanced Network Defense in MANET-IoT Networks. IEEE Access, 2020. 8: pp. 183571183582. https://doi.org/10.1109/access.2020.3029736

[4] Quy, V. K., Nam, V. H., Linh, D. M. et al., A Survey of QoS-aware Routing Protocols for the MANET-WSN Convergence Scenarios in IoT Networks. Wireless Pers Commun, 2021. 120(1):pp. 49-62. https://doi.org/10.1007/s11277-021-08433-Z

[5] Farouk, F., Alkady, Y., Rizk, R., Efficient Privacy-Preserving Scheme for Location Based Services in VANET System. IEEE Access, 2020. 8:pp. 60101-60116. https://doi.org/ $\underline{10.1109 / \text { access. } 2020.2982636}$

[6] Li, J., Li, X., Gao, Y., Gao, Y., and Zhang, R., Dynamic Cloudlet-Assisted Energy-Saving Routing Mechanism for Mobile Ad Hoc Networks. IEEE Access, 2017. 5: pp. 20908-20920. https://doi.org/10.1109/access.2017.2759138.

[7] F. Farouk, Y. Alkady and R. Rizk, Efficient Privacy-Preserving Scheme for Location Based Services in VANET System, IEEE Access, 2020. 8: pp. 60101-60116. https://doi.org/ $\underline{10.1109 / \text { access.2020.2982636 }}$

[8] Quy, V. K., Nam, V. H., Linh, D. M. et al. Communication Solutions for Vehicle Ad-hoc Network in Smart Cities Environment: A Comprehensive Survey. Wireless Pers Commun (2021). https://doi.org/10.1007/s11277-021-09030-w

[9] Mostafa, K. M., Darwish, S. M., and El-Derini, M. N., Adaptive Mechanism for Discovering Internet Gateways in Wireless Networks Using Swarm Intelligence. IEEE Access, 2021. 9:pp. 47294-47302. https://10.1109/ACCESS.2021.3068527

[10] Kumar, R., Misra, M. and Sarje, A. K., An Efficient Gateway Discovery in Ad Hoc Networks for Internet Connectivity, International Conference on Computational Intelligence and Multimedia Applications, 2007. pp. 275-282. https://doi.org/10.1109/iccima.2007.73

[11] Khan, K. U. R., Reddy, A. V., Zaman, R. U. and Kumar, M., An Effective Gateway Discovery Mechanism in an Integrated Internet-MANET (IIM). International Conference on Advances in Computer Engineering, 2010. pp. 24-28. https://doi.org/10.1109/ace.2010.27

[12] Singh, R. and Prakash, J., Gateway Selection Scheme for MANET to Internet Connectivity: A Survey. The 8th International Conference on Reliability, Infocom Technologies and Optimization (Trends and Future Directions) (ICRITO), Noida, India, 2020. pp. 1110-1114. https://doi.org/10.1109/icrito48877.2020.9197892

[13] Z. Hui, Z. Lingli, Y. Yonghang, and C. Linlin, A Review of Gateway Load Balancing Methods in Connecting MANET into Internet. The 23rd International Conference on Advanced Communication Technology (ICACT), PyeongChang, Korea (South), 2021. pp. 330-335. https://doi.org/10.23919/icact51234.2021.9370742

[14] Xu, H., Zhao, Y., Zhang, L., and Wang, J., A Bio-Inspired Gateway Selection Scheme for Hybrid Mobile Ad Hoc Networks. IEEE Access, 2019. 7: pp. 61997-62010. https://doi. org/10.1109/access.2019.2916189

[15] Chen, T. and Wu, B., Gateway Selection based on Game Theory in Internet of Things. International Conference on Electronics Technology (ICET), Chengdu, China, 2018. pp. 403406. https://doi.org/10.1109/eltech.2018.8401412

[16] Shi, Y., Xia, Y. and Gao, Y., Joint Gateway Selection and Resource Allocation for CrossTier Communication in Space-Air-Ground Integrated IoT Networks. IEEE Access, 2021. 9: pp. 4303-4314. https://doi.org/10.1109/access.2020.3047891 
[17] Qin, H., Cao, B., He, J., Xiao, X., Chen, W. and Peng, Y., Cross-Interface Scheduling Toward Energy-Efficient Device-to-Gateway Communications in IoT. IEEE Internet of Things Journal, 2020. 7(3): pp. 2247-2262. https://doi.org/10.1109/jiot.2019.2958612

[18] Banaie, F., Misic, J., Misic, V. B., Yaghmaee Moghaddam, M. H. and Hosseini Seno, S. A., Performance Analysis of Multithreaded IoT Gateway. IEEE Internet of Things Journal, 2019. 6(2): pp. 3143-3155. https://doi.org/10.1109/jiot.2018.2879467

[19] RFC3561. [Online]. Available: https://www.ietf.org/rfc/rfc3561.txt

[20] RFC4728. [Online]. Available: https://www.ietf.org/rfc/rfc4728.txt

\section{Authors}

Le Anh Ngoc is a Director of Swinburne Innovation Space, Swinburne University of Technology (Vietnam). He received his B.S in Mathematics and Informatics from Vinh University and VNU University of Science, respectively. He received a Master's degree in Information Technology from Hanoi University of Technology, Vietnam. He obtained a Ph.D. degree in Communication and Information Engineering from the School of Electrical Engineering and Computer Science, Kyungpook National University, South Korea, in 2009. His general research interests are Embedded and Intelligence Systems, Communication Networks, the Internet of Things, Image/Video Processing, AI \& Big Data Analysis. On these topics, he published more than 40 papers in international journals and conference proceedings. He served as a keynote, TPC member, session chair, and reviewer of international conferences and journals (email: ngocla2@fe.edu.vn).

Nguyen Duy Tan was born in Hung Yen Province, Vietnam, in 1977. He received his M.Sc. degree from Posts and Telecommunications Institute of Technology and received his Ph.D. degree in Communication Engineering from the same University in 2021. Currently, he is a lecturer at Hung Yen University of Technology and Education, Viet Nam. Research Areas include Wireless Communications, Mobile Ad-hoc Computing, High Performance (email: tanndhyvn@gmail.com).

Vu Khanh Quy was born in Hai Duong Province, Vietnam, in 1982. He received a Master degree from the Posts and Telecommunications Institute of Technology, Vietnam, in 2012 and obtained the Ph.D. degree in telecommunication engineering at the same university in 2021. Currently, he is a lecturer at the Faculty of Information Technology, Hung Yen University of Technology and Education. Research Areas: Wireless Communications, Mobile Ad-hoc Computing, High Performance, Next-Generation Networks, Edge Computing and Smart Agriculture (https://orcid.org/0000-0002-0242$\underline{5606)}$

Article submitted 2021-05-28. Resubmitted 2021-09-19. Final acceptance 2021-09-19. Final version published as submitted by the authors. 